Ana Luiza d'Ávila Viana ${ }^{1}$

Luiza S. Heimann 2

Luciana Dias de Lima 3

Roberta Gondim de Oliveira 4

Sergio da Hora Rodrigues 5

\title{
Mudanças significativas no processo de descentralização do sistema de saúde no Brasil
}

\author{
Significant changes in the health system \\ decentralization process in Brazil
}

1 Departamento de Medicina Preventiva, Faculdade de Medicina, Universidade de São Paulo. Av. Dr. Arnaldo 455, São Paulo, SP 01246-903, Brasil. analuivi@zaz.com.br 2 Núcleo de Investigação em Serviços e Sistemas de Saúde, Instituto de Saúde, Secretaria de Estado da Saúde de São Paulo. Rua Santo Antônio 590, São Paulo, SP 01314-000, Brasil. luizash@isaude.sp.gov.br

3 Departamento de Administração e Planejamento em Saúde, Escola Nacional de Saúde Pública, Fundação Oswaldo Cruz.

Rua Leopoldo Bulhões 1480, Rio de Janeiro, $R J$ 21041-210, Brasil. luciana@ensp.fiocruz.br 4 Programa de Educação a Distância, Escola Nacional de Saúde Pública, Fundação Oswaldo Cruz. Rua Leopoldo Bulhões 1480, Rio de Janeiro, $R J$ 21041-210, Brasil. betagondim@domain.com.br 5 Núcleo de Estudos de Políticas Públicas, Universidade Estadual de Campinas. Av. Albert Einstein 1300, Campinas, $S P$ 13083-970, Brasil.

sergio@nepp.unicamp.br
Abstract This article discusses the trends and limits of the Brazilian health system decentralization process, identifying the three elements that constitute the strategic induction performed by the national system administrator in accordance with the guidelines contained in the Operational Norms of the Unified National Health System: systemic rationality, intergovernmental and service provider financing, and health care model. The effects of the Federal regulations are analyzed based on the results of the evaluation study focused on the implementation of the full management scheme at the Municipal level. The decentralization strategy induced by Basic Operational Norm 96 has succeeded in improving institutional conditions, management autonomy, and supply, as measured by the Federal resources transferred, installed capacity, production, and coverage of outpatient and hospital services, with the Municipalities authorized to conduct fully autonomous management, without altering the existing patterns of inequity in the distribution of funds to poorer Municipalities.

Key words Health Policy; Decentralization; Basic Standards for Health Care; Health System; Brazilian Health System

Resumo O artigo discute as tendências e os limites do processo de descentralização da política de saúde no Brasil, identificando os três elementos constitutivos da indução estratégica conduzida pelo gestor nacional, nos preceitos das Normas Operacionais do SUS: racionalidade sistêmica, financiamento intergovernamental e dos prestadores de serviço e modelo de atenção à saúde. Os efeitos das regulações federais são analisados com base nos resultados da Pesquisa de Avaliação da Instituição da Gestão Plena do Sistema Municipal. A estratégia de descentralização, induzida pela Norma Operacional Básica 96, vem conseguindo melhorar as condições institucionais, de autonomia gerencial e de oferta - aferidas pelos recursos financeiros federais transferidos, capacidade instalada, produção e cobertura dos serviços ambulatoriais e hospitalares - nos sistemas de saúde dos municípios habilitados em gestão plena, sem alterar os padrões de iniqüidade existentes na distribuição dos recursos para os municípios mais carentes.

Palavras-chave Política de Saúde; Descentralização; Normas Básicas de Atenção à Saúde; Sistema de Saúde; Sistema Único de Saúde 


\section{Apresentação}

Segundo Freeman \& Moran (2000), em edição recente, as atuais transformações nos sistemas nacionais de saúde foram guiadas pelos fortes elos entre as três dimensões da política de saúde: a saúde como proteção social (welfare dimension), como poder político e como mercado econômico (industrial dimension).

Assinale-se a grande contradição observada pelos autores: a face política (saúde como direito) opõe-se aos cortes dos benefícios, e a face econômica, ao declínio das taxas de lucro, em um período dominado pelo imperativo fiscal - corte dos gastos e despesas estatais. Por isso, as reformas encaminharam-se para mudanças organizadoras - na gestão, na competitividade e na qualidade -, buscando combinar macro e microeficiências.

A descentralização de par com o aumento da competitividade através da criação dos mercados internos (paramercados ou mercados administrados) foram dois grandes instrumentos das políticas de reforma. Ressalta-se que ambas as estratégias (mercantilização e descentralização) são intensamente induzidas e comandadas pelos governos centrais, por meio da criação tanto de instrumentos, normas e ações públicas, quanto de capacidades gerenciais.

Portanto, no momento de hegemonia da economia liberal e das propostas de flexibilização e corte dos gastos estatais, o papel do Estado nos sistemas de saúde é paradoxalmente fortificado por meio das funções reguladoras e reordenadoras dos complexos sistemas de provisão dos serviços. O que se pergunta é se esse novo tipo de intervenção propiciou ganhos de eficiência e manteve os compromissos de eqüidade nos sistemas de proteção erguidos na segunda metade do século 20 .

No caso brasileiro, da mesma forma, a política federal nos anos de 1990 se concentrou na indução e na criação de condições para reconstituir responsabilidades gerenciais regionais ou locais. O propósito central desse texto é, justamente, fundado na discussão dos resultados preliminares da Pesquisa de Avaliação da Gestão Plena do Sistema Municipal (GPSM) pesquisa financiada pelo Banco Mundial no âmbito do Projeto REFORSUS (Reforço à Reorganização do Sistema Único de Saúde) e coordenada pelo Departamento de Descentralização da Gestão da Assistência, Secretaria de Assistência à Saúde, Ministério da Saúde (DGA/ SAS /MS) -, extrair lições do caso da reforma brasileira, de forma a reconstituir o trade-off entre a eqüidade e a eficiência no processo de descentralização setorial.

\section{Breve caracterização da problemática da descentralização em saúde}

Há mais de duas décadas, o Brasil vem redefinindo o perfil do sistema de saúde. Nos anos de 1990, reformulam-se os papéis e as funções dos entes governamentais na oferta de serviços, na gerência de unidades e na gestão do sistema de saúde.

A emergência do SUS faz parte de um processo maior, no qual dois importantes fatores merecem destaque: (1) as sucessivas mudanças na política de saúde postas em prática pelas Ações Integradas de Saúde (AIS) e pela introdução do Sistema Unificado e Descentralizado de Saúde (SUDS), já no contexto da democratização e do processo de reforma sanitária (Teixeira, 1990); (2) o processo de territorialização das políticas públicas, impulsionado pelo movimento de urbanização típica dos anos 1970, que induziu os municípios a assumir, de forma aleatória, uma série de responsabilidades e atribuições no campo das políticas sociais e, especificamente da saúde, cuja face política foi o movimento municipalista forte.

Esse novo modelo de sistema de saúde, organizado de forma regionalizada e hierarquizada, sob comando único em cada esfera de governo, segundo as diretrizes da descentralização administrativa e operacional, do atendimento integral à saúde e da participação da comunidade, visando ao controle social, se concretiza somente através do estabelecimento de relações interinstitucionais, intergovernos e interserviços (Viana, 1995).

A descentralização associada à diretriz da gestão única resulta em três arranjos formais para o sistema de saúde: os sistemas municipais, estaduais e o nacional. Mas a integralidade e a hierarquização induzem à formação de outros subsistemas, como conseqüência da discussão intergestores. Admitindo múltiplos partícipes, essas composições são fruto de acordos políticos e do desenvolvimento de instrumentos técnico-operacionais necessários à integração e à manutenção da unicidade do sistema.

Dessa forma, na década de 1990, o governo federal edita sucessivas Normas Operacionais Básicas (NOBs) - portarias ministeriais -a fim de instruir e regular a nova organização do sistema, cujos conteúdos abrangem: definição das formas de transferência de recursos interinstâncias de governo; definição das modalidades de pagamento dos serviços de saúde; instrução do processo de descentralização e de construção de uma rede de serviços capaz de operar com racionalidade sistêmica; definição dos tipos de assistência (básica, de média e alta com- 
plexidade) na oferta e definição do modelo de atenção, que articula ações de promoção, prevenção, cura e recuperação (Levcovitz et al., 2001).

Todavia, o cenário dos anos 1990 não é favorável a mudanças desse teor, pois que as relações intergovernamentais nesse período são marcadas por incessantes conflitos e disputas sobre recursos, centralização ainda excessiva, fragmentação institucional, capacidade reguladora incipiente e com pouca tradição participativa da sociedade (Draibe, 1997). Nesse momento, deve-se indagar se é possível a convivência pacífica de uma política cujo perfil incorpora uma proposta acentuadamente descentralizadora e, a um só tempo, exige pacto federativo mais cooperativo, como o SUS, e o próprio perfil de atuação estatal no Brasil.

De pronto não se pode assegurar que as políticas de descentralização no setor da saúde desencadeiem um círculo virtuoso, pois se sabe que as partilhas regionais e locais giram em torno de recursos reconhecidamente escassos. Dessa forma, mantêm-se as desigualdades de representação e de poder, o que pode intensificar o jogo predatório nas relações entre os entes da Federação nas políticas sociais. Portanto, a Federação não pode prescindir de poderosos recursos reguladores, que devem abarcar estratégias, instrumentos e mecanismos claros de accountability.

Seguindo esse raciocínio, a pergunta a ser feita é: as variáveis internas setoriais - as NOB, e mais recentemente a Norma Operacional da Assistência à Saúde (NOAS) (MS, 2001) - e as variáveis externas extra-setoriais, como as policy making de cada Estado ou município, cumpriram papel neutralizador das influências em ordem macro ou aprofundaram essas mesmas características?

Análises recentes do processo de descentralização na área da saúde apontam a tendência de as variáveis microinstitucionais (poderes locais) terem respondido de forma mais direta pelo (in)sucesso de determinadas políticas, ressaltando um grau de autonomia da gestão local e possibilidade de mudanças (Heimann, 1998).

As respostas às pressões por maior participação dos municípios na área social, assim como a alta heterogeneidade entre eles, propiciaram a formação de modelos singulares de saúde, tanto regionais como locais, e suas configurações dependem de variáveis como: formas anteriores de gestão e organização dos serviços; arranjos políticos e as formas de expressão dos interesses no plano regional; tipo de relacionamento estabelecido entre as esferas de poder-
Executivo, Legislativo e Judiciário; grau de associativismo e de capital social criados e desenvolvidos; perfis de financiamento e gasto local em saúde.

São a qualidade e a natureza desses perfis de atuação, ou ainda, as características do poder municipal que melhor respondem pelo desempenho da implantação de determinadas políticas setoriais.

\section{Limites e possibilidades das Normas Operacionais Básicas do SUS}

A política descentralizadora na área da saúde contou, no Brasil, com uma indução estratégica de centro, através da formulação e da instituição de instrumentos reguladores próprios as NOBs de 1991/1992, de 1993 e de 1996 e a NOAS, nas versões 2001 e 2002.

As NOBs não representam apenas estratégias de indução ou de constrangimento do processo de descentralização; ao contrário, passam a configurar outros espaços de pactuação de interesses na área da saúde, originando ordenamentos, além da emergência e do fortalecimento de novos atores, pela incorporação de inúmeros centros de poder na arena decisória da política. Assinale-se que esse processo de crescente democratização e politização conseqüentes da descentralização faz que a política adquira maior estabilidade, para enfrentar coalizões anti-reformas e a aumentar a capacidade de regulação do sistema de saúde.

Quando analisamos todas as NOBs dos anos 1990, em conjunto, percebe-se que formam um conjunto contínuo, visto que cada norma criou uma série de contradições que passaram a ser resolvidas pela Norma subseqüente, numa tentativa ascendente de adequar o processo de descentralização setorial à racionalidade sistêmica, aos modelos de atenção à saúde e ao financiamento.

São as contradições nessa tríade que conformam, geram tensão e desatualizam cada NOB em operação, como pode ser visto, resumidamente, na Tabela 1.

Observe-se que a instrução de ganhos sistêmicos ao SUS não consta nas primeiras NOBs e aparece somente de forma clara na última deliberação. Na questão do financiamento federal, também se percebe um aperfeiçoamento dos critérios, no sentido de diferir serviços de instâncias governamentais e diversificar os critérios de alocação (combinação produção/ per capita). Quanto ao modelo de assistência, somente no final dos anos 1990 é que se aceleram os incentivos para o privilégio da atenção 
Tabela 1

Elementos constitutivos da regulação do processo de descentralização: racionalidade sistêmica,

financiamento federal e modelos de atenção à saúde.

\begin{tabular}{|c|c|c|c|}
\hline Normas & Racionalidade sistêmica & Financiamento federal & Modelos de atenção \\
\hline NOB 91/92 & Ausente & $\begin{array}{l}\text { - Repasse direto ao prestador, } \\
\text { segundo produção aprovada }\end{array}$ & - Ausente \\
\hline NOB 93 & $\begin{array}{l}\text { Fraca: vinculada às iniciativas } \\
\text { e discussões municipais isoladas }\end{array}$ & $\begin{array}{l}\text { - Repasse direto ao prestador, } \\
\text { segundo produção aprovada } \\
\text { - Transferência fundo a fundo, segundo } \\
\text { montante definido no teto financeiro }\end{array}$ & $\begin{array}{l}\text { - Define responsabilidade sobre algumas } \\
\text { ações programáticas e de vigilância } \\
\text { (sanitária e epidemiológica) para a } \\
\text { modalidade semiplena }\end{array}$ \\
\hline NOB 96 & $\begin{array}{l}\text { Moderada: vinculada às iniciativas } \\
\text { e às discussões intermunicipais, com } \\
\text { participação e intermédio da instância } \\
\text { estadual (PPI) }\end{array}$ & $\begin{array}{l}\text { - Repasse direto ao prestador, } \\
\text { segundo produção aprovada } \\
\text { - Transferência fundo a fundo, segundo } \\
\text { montante definido no teto financeiro } \\
\text { - Transferência fundo a fundo, segundo } \\
\text { valor per capita } \\
\text { - Transferência fundo a fundo, segundo } \\
\text { critérios definidos por programas } \\
\text { específicos }\end{array}$ & $\begin{array}{l}\text { - Programa de Agentes Comunitários de } \\
\text { Saúde/Programa Saúde da Família } \\
\text { - Programas e projetos prioritários para } \\
\text { controle de doenças e agravos (carências } \\
\text { nutricionais, catarata, varizes, atenção } \\
\text { de urgência/emergência, doenças } \\
\text { infecciosas, vigilância sanitária, atenção } \\
\text { á população indígena) }\end{array}$ \\
\hline $\begin{array}{l}\text { NOAS } 2001 \\
\text { e } 2002\end{array}$ & $\begin{array}{l}\text { Forte: vinculada às definições do } \\
\text { conjunto de ações e serviços a ser } \\
\text { abrangidos nos módulos assistenciais } \\
\text { pela esfera federal e às iniciativas } \\
\text { e discussões intermunicipais sob } \\
\text { coordenação da instância estadual } \\
\text { (PPI, PDR e PDI) }\end{array}$ & $\begin{array}{l}\text { - Repasse direto ao prestador, } \\
\text { segundo produção aprovada } \\
\text { - Transferência fundo a fundo, segundo } \\
\text { montante definido no teto financeiro } \\
\text { - Transferência fundo a fundo, segundo } \\
\text { valor per capita } \\
\text { - Transferência fundo a fundo, segundo } \\
\text { critérios definidos por programas } \\
\text { específicos } \\
\text { - Transferência fundo a fundo, segundo } \\
\text { valor per capita e definição de } \\
\text { referências intermunicipais }\end{array}$ & $\begin{array}{l}\text { - Mantém o definido na NOB } 96 \text { e } \\
\text { - Derescenta: } \\
\text { e conteção das responsabilidades mínimas } \\
\text { - Redefinição de procedimentos da } \\
\text { atenção de média complexidade } \\
\text { - Redefinição de procedimentos da } \\
\text { atenção de alta complexidade } \\
\text { - Criação de protocolos para assistência } \\
\text { médica }\end{array}$ \\
\hline
\end{tabular}

básica e para a conversão do antigo modelo de assistência (cujas características básicas são enfatizar as ações curativas e ser liderado pela assistência hospitalar).

E o esforço para consolidar um determinado processo de descentralização do sistema de saúde, pautado pela necessidade de compensar as diferenças locais, numa descentralização menos heterogênea, orientou a formulação e a introdução de alguns instrumentos-chave, incluídos nas NOBs do SUS, como: (1) a criação e a ampliação das funções dos Conselhos de Saúde e das Comissões Intergestores Tripartite e Bipartite (CIT e CIB); (2) as transferências automáticas per capita - Piso de Atenção Básica (PAB) - fixo e variável; (3) os requisitos e incentivos ao desenvolvimento e à assunção de capacidades gestoras (aprendizado institucional), vinculados ao processo de habilitação, cujas modalidades estão previstas nas NOBs 93 e 96, para municípios e estados.

A despeito de a NOB 96 ter previsto um conjunto de responsabilidades e regras para os mu- nicípios habilitarem-se nas diferentes condições de gestão, a decisão sobre o processo de habilitação e, particularmente, sobre a partilha de gestão da rede de serviços locais entre estado e municípios foi atribuída às CIBs.

Disposições como essa permitiram a geração dos mais variados tipos ou modelos de gestão e partilha entre as diversas unidades da Federação. Há, portanto, graus variados de poder de interferência do gestor municipal em seu território, nem sempre diretamente associado à condição de habilitação ou gestão prevista na NOB 96. Essas diferentes composições refletem, principalmente, as particularidades do relacionamento e os acordos entre as instâncias de governo estadual e municipal.

Em dezembro de 1998, 4.597 municípios estavam habilitados em gestão plena da atenção básica (83\%) e 452, em GPSM (8,2\%). Em dezembro de 2000, 99\% dos municípios estavam habilitados em alguma condição de gestão, sendo 523 municípios em gestão plena do sistema $(9,4 \%)$. O processo de habilitação dos 
Estados foi mais lento, e, em dezembro de 2000, quatro estados estavam habilitados em gestão plena do sistema estadual e quatro, em gestão avançada.

As transferências automáticas (fundo a fundo) descentralizaram os recursos da saúde, alcançando milhares de municípios que, até então, não haviam sido contemplados com repasses federais, e também estimulou maior autonomia ao gestor municipal. Segundo dados do MS, em dezembro de 2000, 5.450 municípios (99\%) e oito Estados já recebiam recursos fundo a fundo, representando cerca de $61 \%$ do total de recursos federais para custeio da assistência.

O incentivo ao aprendizado institucional, implícito nas modalidades de habilitação gradativa (incipiente parcial semiplena na NOB 93; gestão plena da atenção básica e GPSM na NOB 96), constitui importante instrumento para a melhoria da qualidade da gestão pública.

Estudos recentes, como o que será apresentado a seguir, têm apontado que municípios com trajetória de habilitação, sobretudo os que atingem as modalidades mais qualificadas (como a semiplena na NOB 93 e plena na NOB 96), apresentam melhores condições institucionais e administrativas para a gestão pública da saúde. Dessa forma, pode-se inferir que o processo de qualificação e a capacidade institucional têm sido relevantes à estratégia induzida de descentralização, na qual o governo central planeja e traça as metas a serem atingidas.

Mas há de se apontar que a complexidade e a diversidade do modelo de gestão do SUS, em conjunção com as imensas desigualdades regionais e com o contexto de relações federativas competidoras e predatórias, tendem a manter a tensão da tríade formada por racionalidade sistêmica, financiamento e modelo de atenção.

\section{Avaliação da gestão plena do sistema municipal}

\section{Desenho metodológico do estudo}

Apesar da acelerada descentralização das responsabilidades de gestão e de recursos financeiros da esfera federal para os municípios, observada nos anos de 1990, não existe, até o momento, uma avaliação global dos resultados desse processo. Tal estudo, em andamento, compreende uma análise da gestão descentralizada do SUS no período de implantação da NOB 96, ou seja, a partir de 1998, com ênfase na avaliação dos 523 municípios habilitados em GPSM até o final de 2000 , bem como o desenho de uma estratégia sistemática de acompanhamento da gestão descentralizada do SUS.

Os municípios habilitados na condição de GPSM, apesar de formalmente iguais perante a NOB 01/96, do ponto de vista de suas responsabilidades e atribuições, são muito desiguais nas condições sócio-econômicas e demográficas, na capacidade fiscal, na trajetória no SUS e nas disponibilidades de recursos de saúde (incluindo recursos financeiros, capacidade instalada, produção e cobertura de ações e serviços). Além disso, como discutido anteriormente, a gestão municipal plena dos recursos de saúde está sujeita aos acertos e às discussões definidos na esfera estadual pelas respectivas instâncias intergestoras (CIB).

A classificação dos 523 municípios em gestão plena do sistema municipal, desenvolvida pela pesquisa, tem por fim estabelecer uma tipologia dos municípios enquadrados nessa modalidade de gestão, a fim de avaliar o grau de interferência dessas características e do processo de habilitação no progresso e no maior adensamento dos sistemas municipais de saúde.

Os propósitos específicos do estudo são: (1) conhecer, de modo sistemático, as características dos municípios em GPSM; (2) conhecer o processo de implantação da GPSM; (3) conhecer a extensão e os modelos de municipalização em saúde desenvolvidos na GPSM, de forma a apreender os tipos desenvolvidos de municipalização, segundo capacidade de gestão municipal; os efeitos dos modelos de municipalização nos resultados da gestão; os benefícios e os limites da municipalização notadamente em relação à integração dos níveis de assistência; os condicionantes do exercício do poder gestor municipal; (4) identificar e sistematizar os fatores institucionais que sujeitam positiva ou negativamente a municipalização em saúde; (5) avaliar comparativamente os municípios em GPSM.

Esse estudo reconhece a importância de se avaliar o processo de instituição da GPSM, produzindo conhecimentos sobre o processo de descentralização em saúde e formulando recomendações de políticas, especialmente para construção da regionalização e de sistemas de saúde, em um país com acentuadas diferenças de ordem regional e sócio-econômica e com diferentes capacidades administrativas/institucionais no plano municipal.

Como variáveis independentes, foram utilizados 156 indicadores referentes às condições da oferta assistencial nos municípios (capacidade instalada, produção, financiamento e gasto) obtidos para 1998 e 2000 , que permitiram a formação de um banco de dados relativo aos 
424 municípios habilitados em GPSM até o final de 1998 e outro incluindo o universo dos 523 municípios habilitados até o final de 2000. As bases de dados utilizadas na construção dos indicadores foram: cadastro de estabelecimentos de saúde ambulatorial e hospitalar (SIA e SIH-SUS); produção ambulatorial e hospitalar (SIA e SIH-SUS); recursos federais do SUS; Sistema de Informações de Orçamentos Públicos em Saúde (SIOPS) e Fundação Instituto Brasileiro de Geografia e Estatística (IBGE).

Os indicadores foram organizados em sete eixos principais: (1) padrão da oferta ambulatorial existente no município - capacidade instalada, produção e cobertura de serviços ambulatoriais; (2) padrão da oferta hospitalar existente no município - capacidade instalada, produção e cobertura de serviços hospitalares; (3) padrão da oferta ambulatorial sob gestão municipal - capacidade instalada, produção e cobertura de serviços ambulatoriais sob gestão municipal; (4) padrão da oferta hospitalar sob gestão municipal - capacidade instalada, produção e cobertura de serviços hospitalares sob gestão municipal; (5) padrão do financiamento e gasto federal; (6) padrão do financiamento e gasto municipal; (7) modelo de atenção à saúde adotado no município.

Entende-se, neste estudo, por gestão municipal (ou o termo sob gestão municipal) a autonomia administrativa e financeira do município para o credenciamento, a contratação, a programação, o controle, a avaliação e o pagamento de prestadores de serviços públicos e privados em seu território.

Com base no tratamento estatístico de 54 variáveis representativas das condições de oferta assistencial nos municípios em GPSM, em 1998, foram gerados cinco indicadores sintéticos por meio da utilização da técnica de análise multivariada (factor analysis). Entre as variáveis, sobressaem os tipos de serviços existentes (ambulatorial e hospitalar), o nível de complexidade dos serviços ambulatoriais produzidos (básica, média e alta complexidade), o financiamento federal e o grau de autonomia de gestão municipal.

Essa técnica estatística tem como propósito justamente reduzir o número de variáveis selecionadas para a análise, transformando-as em componentes de indicadores compostos. Uma redução do número de variáveis foi realizada, sem uma perda significativa de informação, já que os indicadores gerados pela análise fatorial são combinações lineares das variáveis originais. A diferença desse tipo de técnica em relação aos métodos usuais de criação de indicadores é que, em tal caso, os "pesos" de cada um dos componentes do indicador composto não são arbitrados de forma subjetiva pelo pesquisador, mas obtidos pelos próprios dados da análise fatorial.

Deve-se destacar, portanto, que, entre as 54 variáveis tratadas originariamente, nem todas foram utilizadas no modelo em razão de não contribuírem significativamente para a explicação da variabilidade dos dados, de estarem muito correlacionadas com outras variáveis do modelo ou de se ter verificado para elas ausência de valores. Um exemplo dessa situação é o valor médio da internação em hospitais públicos em municípios onde não existe tal tipo de hospital. As variáveis utilizadas no estudo são apresentadas na Tabela 2 .

Os cinco fatores gerados pela análise multivariada foram:

- Fator 1 - Capacidade Municipal em Média Complexidade: este fator expressa a produção de procedimentos de média complexidade, segundo a classificação da Tabela de Procedimentos do SIA-SUS. Valores altos nesse indicador significam maior produção de procedimentos de média complexidade no total e sob gestão municipal.

- Fator 2 - Capacidade Municipal em Atenção Básica: neste fator, as maiores correlações estão com as variáveis referentes aos procedimentos de atenção básica. Analogamente ao fator 1, altos valores nesse indicador implicam maior produção e cobertura de procedimentos de atenção básica no total e sob gestão municipal. - Fator 3 - Capacidade Hospitalar: expressa o número de internações totais e a participação dos não-residentes (ou de residentes em outros municípios) no total das internações realizadas no município, independentemente da capacidade de gestão municipal.

- Fator 4 - Capacidade Municipal em Alta Complexidade: expressa a produção e cobertura de procedimentos de alta complexidade. Valores altos nesse indicador significam maior produção e cobertura de procedimentos de alta complexidade no total e sob gestão municipal. - Fator 5 - Capacidade Municipal Hospitalar: expressa a capacidade de gestão do município em relação à atenção hospitalar e o volume de recursos financeiros federais transferidos.

Observe-se que as mesmas variáveis utilizadas na criação dos agrupamentos em 1998 foram atualizadas para 2000.

Posteriormente, com a finalidade de identificar grupos homogêneos de municípios nos anos selecionados, utilizou-se a técnica de análise de agrupamentos (cluster analysis). Essa técnica permitiu a geração de seis grupos ou tipos de municípios em GPSM diferenciados pe- 
Variáveis utilizadas no modelo fatorial da Pesquisa de Avaliação da Gestão Plena do Sistema Municipal.

\begin{tabular}{ll}
\hline Descrição & Unidade \\
\hline \% de internações em hospitais sob gestão municipal no total de internações realizadas & em porcentagem \\
Número de internações em hospitais sob gestão municipal por habitantes & por habitante \\
Transferências federais de MC e AC por habitante & em Reais por habitante \\
Número de ações de média complexidade por mil habitantes & em mil habitantes \\
Número de ações de média complexidade por habitantes sob gestão municipal & em mil habitantes \\
\% de ações básicas no total de atendimentos em unidades públicas & em porcentagem \\
\% de atendimentos básicos no total de atendimentos sob gestão municipal & em porcentagem \\
Número de ações básicas por habitantes sob gestão municipal & em mil habitantes \\
Número de ações básicas por mil habitantes & em mil habitantes \\
Transferências federais de atenção básica por habitante & em Reais por habitante \\
Número de ações de alta complexidade por mil habitantes & em mil habitantes \\
Número de ações de alta complexidade por habitantes sob gestão municipal & em mil habitantes \\
Número de internações por habitantes & por habitante \\
\hline
\end{tabular}

Fonte: Departamento de Informática do SUS.

las condições de oferta assistencial em 1998 e 2000 e descritos segundo o local (estado/região), o porte populacional, o aprendizado institucional, a capacidade de gasto municipal medido pelo índice de capacidade de gasto e da receita de orçamento per capita, o percentual de gasto com pessoal no gasto total municipal e a classificação sócio-econômica e demográfica desenvolvida pelo Núcleo de Economia Social, Urbana e Regional da Universidade Estadual de Campinas (NESUR/UNICAMP).

$\mathrm{O}$ aprendizado institucional do município foi medido através de sua trajetória de habilitação nas NOB 93 e NOB 96. Foram considerados como tendo aprendizado institucional baixo os municípios que passaram diretamente para a GPSM (NOB 96) ou da condição de gestão plena da atenção básica (NOB 96) para a plena (NOB 96), sem apresentarem trajetória de habilitação na NOB 93. Como aprendizado institucional médio, foram classificados os municípios que passaram da condição de gestão incipiente ou parcial (NOB 93) para plena (NOB 96), os municípios que passaram de incipiente ou parcial, para plena da atenção básica e depois para a plena (NOB 96). Os municípios considerados como tendo alto aprendizado institucional foram habilitados na modalidade de gestão mais avançada da NOB 93 antes da plena (NOB 96), isto é, passaram pela condição de gestão semiplena (NOB 93). Excetuam-se, neste caso, os municípios que passaram da condição de gestão semiplena (NOB 93), para plena da atenção básica e posteriormente plena (NOB
96). Estes tiveram um médio aprendizado institucional, pois apresentaram perda na autonomia de gestão, na passagem da NOB 93 para a NOB 96.

Quanto à classificação NESUR utilizada, aí são considerados o porte e a dinâmica sócioeconômica dos municípios através dos seguintes indicadores: (1) tamanho da população; 2) percentual da população economicamente ativa (PEA) ocupada em agricultura, indústria, comércio e serviços auxiliares da atividade econômica; (3) percentual da PEA ocupada em atividades administrativas e técnicas; (4) renda média familiar per capita; (5) índice de consumo de bens nos domicílios particulares urbanos (telefone, automóvel, rádio, refrigerador, TV, freezer, máquina de lavar); (6) anos médios de estudo da população (para pessoas maiores de cinco anos de idade); (7) índice de infra-estrutura urbana (medido pelo percentual de domicílios com abastecimento de água adequado, com rede geral de esgoto ou fossa séptica, com lixo coletado e com abastecimento de energia elétrica). Esses indicadores são combinados com um critério de classificação pelo qual o município pode ser: (a) pequeno, (b) médio, (c) grande, (d) sedes de região metropolitana ou capitais e (e) periferia de região metropolitana, de acordo com sua inserção na malha urbana. A classificação final dos municípios define: pequenos, médios, grandes, periferia de regiões metropolitanas e capital.

Outra variável independente desenhada foi o processo de instituição da gestão do sistema 
municipal, medido por sete blocos de questões referentes ao modelo de gestão, planejamento, financiamento, recursos humanos e de atenção à saúde adotados pelos municípios e a trajetória do processo de habilitação. Para tanto, foi elaborado um questionário auto-aplicável para o universo dos municípios estudados.

A variável dependente selecionada referese ao desempenho e aos resultados da gestão municipal em saúde. Os resultados da gestão foram assimilados com a variedade das condições de oferta e de autonomia gerencial dos sistemas municipais de saúde, o que denominamos capacidade de progresso e adensamento dos sistemas municipais de saúde (variação cluster 1998-2000). Neste artigo, como resultados preliminares da pesquisa, será apresentada a análise dos agrupamentos de municípios e da variação observada nos grupos no período de 1998 a 2000.

\section{Composição, expansão/retração e migração intergrupos de municípios no período de 1998 a 2000}

Como já foi tratado anteriormente, utilizou-se a técnica de análise de agrupamentos com a finalidade de identificar grupos homogêneos de municípios, segundo os cinco fatores gerados pela análise fatorial. Os municípios habilitados em GPSM foram então agrupados com base nas médias apresentadas por cada um nos fatores.

Resumidamente, podem-se apresentar os seis agrupamentos finais como tendo as seguintes características:

- Grupo 1 - Alta capacidade de oferta municipal na atenção básica: composto por municípios que têm a maior média do fator 2 (produção e cobertura em atenção básica) e médias baixas em todos os outros fatores. Nos fatores 3, 4 e 5 apresentam as menores médias.

- Grupo 2 - Alta capacidade de oferta municipal em alta complexidade: composto por municípios que têm a maior média para o fator 4 , isto é, maior capacidade de produção e cobertura em alta complexidade. Todos os outros fatores apresentam também médias altas.

- Grupo 3 - Alta capacidade de oferta hospitalar: composto por municípios que apresentam a maior média no fator 3 (produção e cobertura hospitalar), médias altas nos fatores 1 (média complexidade) e 2 (atenção básica) e médias baixas nos fatores 4 (alta complexidade) e 5 (gestão hospitalar).

- Grupo 4 - Alta capacidade de oferta municipal hospitalar com maiores transferências federais per capita: engloba os municípios que apresentam a maior média no fator 5 (capaci- dade de gestão hospitalar e maiores volumes de recursos financeiros federais transferidos) e a menor média no primeiro fator (média complexidade), tendo médias baixas nos fatores 2 (atenção básica) e 3 (produção e cobertura hospitalar).

- Grupo 5 - Baixa capacidade de oferta e de gestão municipal: constituído por municípios que têm todos as médias negativas, sendo a média do fator 2 (atenção básica) a menor de todos os grupos.

- Grupo 6 - Alta capacidade de oferta municipal em média complexidade: engloba municípios que têm a maior média do fator 1 (produção e cobertura em média complexidade) e os demais fatores abaixo da média global, exceto no fator 5 (capacidade de gestão hospitalar).

Na Tabela 3, apresentamos o perfil de cada agrupamento para os anos de 1998 e 2000, segundo a região, o porte populacional, o aprendizado institucional, a receita de orçamento per capita, a capacidade de gasto e o gasto com pessoal.

Fundado na análise do comportamento dos municípios nos agrupamentos em 1998 e 2000, pode-se observar a existência de acentuado movimento de expansão/retração dos grupos cujas características ressaltam: (1) expansão de 1,5 vez do grupo de alta capacidade de oferta municipal em média complexidade (153\%); (2) expansão de quase 1,0 vez no grupo de alta capacidade de oferta municipal em alta complexidade (92\%); (3) expansão de $61 \%$ do grupo de alta capacidade de oferta municipal na atenção básica; (4) diminuição em quase $90 \%$ do grupo de baixa capacidade de oferta e de gestão municipal; (5) diminuição de quase $40 \%$ do grupo de alta capacidade de oferta hospitalar.

O grau de permanência dos municípios nos grupos, em ordem decrescente, é caracterizado por manutenção de $88 \%$ dos municípios em 1998 no grupo alta capacidade de oferta em alta complexidade (grupo 2); manutenção de $47 \%$ dos municípios no grupo alta capacidade de oferta municipal em média complexidade (grupo 6); manutenção de 18\% dos municípios no grupo alta capacidade de oferta hospitalar sob gestão municipal com maiores transferências federais per capita; manutenção de $8 \%$ dos municípios no grupo alta capacidade de oferta na atenção básica (grupo 1) e manutenção de $4 \%$ dos municípios no grupo baixa capacidade de oferta e de gestão municipal (grupo 5). Todos os municípios do grupo alta capacidade de oferta hospitalar (grupo 3) em 1998 não permaneceram no mesmo grupo em 2000.

O perfil de migração intergrupos, responsável pela saída e pela entrada de municípios en- 
Perfil dos agrupamentos de municípios segundo algumas variáveis descritivas utilizadas no estudo - 1998 e 2000 .

\begin{tabular}{|c|c|c|c|c|c|c|c|c|c|c|}
\hline \multirow[t]{3}{*}{ Grupo } & \multicolumn{4}{|c|}{ Municípios } & \multicolumn{2}{|c|}{ População (\%) } & \multicolumn{2}{|c|}{ Região } & \multicolumn{2}{|c|}{$\begin{array}{c}\text { Municípios até } \\
50.000 \text { habitantes (\%) }\end{array}$} \\
\hline & \multicolumn{2}{|c|}{1998} & \multicolumn{2}{|c|}{2000} & \multirow[t]{2}{*}{1998} & \multirow[t]{2}{*}{2000} & \multirow[t]{2}{*}{1998} & \multirow[t]{2}{*}{2000} & \multirow[t]{2}{*}{1998} & \multirow[t]{2}{*}{2000} \\
\hline & $\mathrm{N}$ & $\%$ & $\mathrm{~N}$ & $\%$ & & & & & & \\
\hline $\begin{array}{l}\text { Alta capacidade de oferta } \\
\text { municipal na atenção básica }\end{array}$ & 75 & 18 & 121 & 23 & 15 & 8 & $\begin{array}{l}95 \%- \\
\text { Sudeste }\end{array}$ & $\begin{array}{l}36 \%- \\
\text { Nordeste } \\
\text { e 33\% - } \\
\text { Sudeste }\end{array}$ & 51 & 80 \\
\hline $\begin{array}{l}\text { Alta capacidade de oferta } \\
\text { municipal em alta } \\
\text { complexidade }\end{array}$ & 40 & 9 & 77 & 15 & 37 & 53 & $\begin{array}{l}60 \%- \\
\text { Sudeste } \\
\text { e } 22 \%- \\
\text { Centro-Oe }\end{array}$ & $\begin{array}{l}48 \%- \\
\text { Sudeste }\end{array}$ & 0 & 10 \\
\hline $\begin{array}{l}\text { Alta capacidade de oferta } \\
\text { hospitalar }\end{array}$ & 46 & 11 & 29 & 6 & 7 & 3 & $\begin{array}{l}57 \%- \\
\text { Sudeste } \\
\text { e } 33 \%- \\
\text { Nordeste }\end{array}$ & $\begin{array}{l}55 \%- \\
\text { Sudeste } \\
\text { e } 35 \%- \\
\text { Nordeste }\end{array}$ & 65 & 52 \\
\hline $\begin{array}{l}\text { Alta capacidade de oferta } \\
\text { municipal hospitalar com } \\
\text { maiores transferências federais } \\
\text { per capita }\end{array}$ & 89 & 21 & 95 & 18 & 13 & 7 & $\begin{array}{l}56 \%- \\
\text { Sudeste } \\
\text { e } 23 \%- \\
\text { Nordeste }\end{array}$ & $\begin{array}{l}39 \%- \\
\text { Nordeste } \\
\text { e 30\% - } \\
\text { Sudeste }\end{array}$ & 50 & 75 \\
\hline $\begin{array}{l}\text { Baixa capacidade de oferta } \\
\text { e de gestão municipal }\end{array}$ & 99 & 23 & 11 & 2 & 12 & 1 & $\begin{array}{l}47 \%- \\
\text { Sudeste } \\
\text { e } 27 \%- \\
\text { Nordeste }\end{array}$ & $\begin{array}{l}55 \%- \\
\text { Nordeste } \\
\text { e } 27 \%- \\
\text { Sudeste }\end{array}$ & 60 & 73 \\
\hline $\begin{array}{l}\text { Alta capacidade de oferta } \\
\text { municipal em média } \\
\text { complexidade }\end{array}$ & 75 & 18 & 190 & 36 & 16 & 29 & $\begin{array}{l}35 \%- \\
\text { Sudeste } \\
\text { e } 33 \%- \\
\text { Nordeste }\end{array}$ & $\begin{array}{l}76 \%- \\
\text { Sudeste }\end{array}$ & 41 & 45 \\
\hline \multirow[t]{2}{*}{ Grupo } & \multicolumn{4}{|c|}{$\begin{array}{l}\text { Aprendizado institucional } \\
\text { alto (\%) }\end{array}$} & \multicolumn{2}{|c|}{$\begin{array}{c}\text { Receita de orçamento } \\
\text { per capita (R\$) }\end{array}$} & \multicolumn{2}{|c|}{ Capacidade de gasto $\left(^{*}\right)$} & \multicolumn{2}{|c|}{ Gasto com pessoal (\%) } \\
\hline & 1998 & & 20 & & 1998 & 2000 & 1998 & 2000 & 1998 & 2000 \\
\hline $\begin{array}{l}\text { Alta capacidade de oferta } \\
\text { municipal na atenção básica }\end{array}$ & 8 & & & & 420,38 & 230,62 & 0,44 & 0,22 & 51 & 37 \\
\hline $\begin{array}{l}\text { Alta capacidade de oferta } \\
\text { municipal em alta } \\
\text { complexidade }\end{array}$ & 40 & & 3 & & 388,99 & 320,91 & 0,40 & 0,22 & 49 & 47 \\
\hline $\begin{array}{l}\text { Alta capacidade de oferta } \\
\text { hospitalar }\end{array}$ & 65 & & 1 & & 471,97 & 263,66 & 0,30 & 0,30 & 45 & 48 \\
\hline $\begin{array}{l}\text { Alta capacidade de oferta } \\
\text { municipal hospitalar com } \\
\text { maiores transferências federais } \\
\text { per capita }\end{array}$ & 5 & & 3 & & 264,75 & 237,80 & 0,33 & 0,27 & 48 & 42 \\
\hline $\begin{array}{l}\text { Baixa capacidade de oferta } \\
\text { e de gestão municipal }\end{array}$ & 4 & & & & 269,60 & 201,39 & 0,32 & 0,21 & 42 & 33 \\
\hline $\begin{array}{l}\text { Alta capacidade de oferta } \\
\text { municipal em média } \\
\text { complexidade }\end{array}$ & 60 & & 2 & & 291,77 & 405,86 & 0,29 & 0,39 & 43 & 49 \\
\hline
\end{tabular}

Fonte: Pesquisa Avaliação da Gestão Plena do Sistema Municipal, 2001.

(*) Calculado com base no Imposto sobre Serviço (ISS) e no Imposto Predial e Territorial Urbano (IPTU) somados e divididos pela receita de orçamento per capita.

Nota: Agrupamento de 1998: 424 municípios classificados e 99 municípios não classificados. Agrupamento de 2000:

523 municípios classificados. 
tre 1998 e 2000, caracteriza-se, para cada um deles, da seguinte maneira:

- Grupo da alta capacidade de oferta hospitalar (Grupo 3) - foi extinto na sua composição original. Os municípios que passaram a compor esse grupo vieram do grupo de alta capacidade de oferta municipal hospitalar com maiores transferências federais per capita (62\%), do grupo dos não classificados (31\%) e do grupo de alta capacidade de oferta municipal em média complexidade (7\%).

- Grupo de baixa capacidade de oferta e de gestão municipal (Grupo 5) - foi praticamente extinto, e os municípios migraram predominantemente para os grupos de alta capacidade de oferta municipal na atenção básica (42\%) e alta capacidade de oferta municipal hospitalar com maiores transferências federais per capita (30\%). Os municípios que passaram a compor esse grupo vieram dos municípios não classificados em 1998 (36\%) e do grupo de alta capacidade de oferta municipal hospitalar com maiores transferências federais per capita $(27 \%)$.

- Grupo de alta capacidade de oferta municipal na atenção básica (Grupo 1) - migrou majoritariamente para o grupo de alta capacidade de oferta municipal em média complexidade. O atual grupo de atenção básica é formado na sua maior parte pelos municípios que anteriormente estavam no grupo dos municípios não classificados em 1998 (41\%) e que apresentava baixa capacidade de oferta e de gestão municipal (35\%). - Grupo de alta capacidade de oferta municipal hospitalar com maiores transferências federais per capita (Grupo 4) - permaneceram no grupo apenas $20 \%$ dos municípios originais. Migraram 28\% para o grupo de alta capacidade de oferta municipal em média complexidade, $20 \%$ para o grupo de alta capacidade de oferta hospitalar e $19 \%$ para o grupo de alta capacidade de oferta municipal na atenção básica. Vieram compor esse grupo $34 \%$ dos municípios que compunham o grupo de alta capacidade de oferta municipal em média complexidade e $15 \%$ do grupo de alta capacidade de oferta hospitalar. - Grupo de alta capacidade de oferta municipal em média complexidade (grupo 6) - foi o de maior expansão, tendo permanecido $47 \%$ dos municípios originais e migrado $43 \%$ para o grupo de capacidade instalada hospitalar sob gestão municipal com maiores transferências federais per capita. Os municípios que passaram a compor esse grupo vieram majoritariamente do grupo dos não classificados em 1998 (36\%), de alta capacidade de oferta municipal na atenção básica (33\%) e de capacidade instalada hospitalar sob gestão municipal com maiores transferências federais per capita (13\%).
- Grupo de alta capacidade de oferta municipal em alta complexidade (Grupo 2) - manteve-se praticamente inalterado $(90 \%)$. Como houve expansão de quase 1,0 vez (90\%), os municípios que passaram a compor esse grupo vieram do grupo dos não classificados em 1998 (17\%) e de alta capacidade de oferta municipal hospitalar com maiores transferências federais per capita $(13 \%)$.

Os municípios não classificados em 1998 foram para os grupos de alta capacidade de oferta municipal na atenção básica (50\%) e de alta capacidade de oferta municipal na média complexidade (23\%).

Os resultados preliminares do estudo indicam que houve significativa mudança no perfil de caracterização dos grupos dos municípios em GPSM, segundo sua capacidade de oferta por níveis de assistência e gestão municipal. Há uma tendência de progresso que segue diferentes trajetos:

- O grupo de alta capacidade de oferta municipal em alta complexidade é estável, no sentido que consegue reter quase que $90 \%$ dos municípios, por um lado; por outro, foi o segundo grupo em expansão.

- O grupo de alta capacidade de oferta municipal na atenção básica evolui para o grupo de alta capacidade de oferta municipal em média complexidade.

- Os grupos de alta capacidade de oferta hospitalar e de capacidade de gestão hospitalar com maiores transferências federais per capita mudam significativamente de perfil sem expandirem-se (o primeiro perde municípios e o segundo cresce muito pouco). Ambos migram para o grupo de alta capacidade de oferta municipal em média complexidade, mas o grupo de capacidade instalada hospitalar com maiores transferências federais per capita, além de migrar para o grupo de alta capacidade de oferta em média complexidade, direciona-se, também, para o grupo de alta capacidade de oferta hospitalar.

- Os municípios do grupo de baixa capacidade de oferta e de gestão municipal evoluem para os grupos de alta capacidade de oferta em atenção básica e de capacidade instalada hospitalar com maiores transferências federais per capita.

- Os municípios do grupo de alta capacidade de oferta municipal em média complexidade comportam-se de forma semelhante, isto é, metade permanece no mesmo grupo e metade migra para o grupo de capacidade de gestão hospitalar com maiores transferências federais per capita. 
Caracterização dos grupos de municípios habilitados em gestão plena do sistema municipal, por níveis de assistência 1998 e 2000

Para análise dos agrupamentos de municípios dos anos 1998 e 2000, segundo níveis assistenciais, foram construídas categorias valorando os fatores considerados para a conformação dos grupos em alto, médio e baixo. Os fatores foram considerados como altos quando seus valores estavam acima da média para $50 \%$ ou mais municípios integrantes do grupo.

A Tabela 4 apresenta um quadro resumo da variação entre 1998 e 2000 dos fatores que também passaram a se destacar na composição dos grupos segundo os níveis de assistência.

Em 1998, somente os grupos 2 e 3 se destacam também em mais de um nível de atenção, além do nível de atenção que os caracteriza (alta oferta municipal em alta complexidade e alta oferta hospitalar). O grupo 2 agrega mais dois níveis de atenção em que o fator correspondente a esse tipo de ação teve igual ou mais de $50 \%$ dos municípios do grupo com avaliação alta, e o grupo 3 apenas um nível mais (atenção ambulatorial de média complexidade). Assinale-se que, em 1998, somente o grupo 2 sobressai também em atenção básica. Em suma, em 1998, o grupo 2 combina três níveis de atenção (alta complexidade, atenção básica e capacidade de gestão hospitalar) e o grupo 3, dois níveis (capacidade de gestão hospitalar e atenção em média complexidade).
Em 2000, observe-se, em primeiro lugar, que todos os grupos, exceto o grupo 5, incorporam a atenção básica. Em segundo, pode-se afirmar que se amplia a abrangência da composição dos níveis de atenção, pois o grupo 2 combina quatro níveis (alta complexidade, atenção básica, capacidade de gestão hospitalar e média complexidade); o grupo 3, três níveis (capacidade de gestão hospitalar, média complexidade e atenção básica) e os grupos 4 e 6, dois níveis (atenção básica e os níveis que os definem - capacidade de gestão hospitalar e média atenção ambulatorial).

Pode-se afirmar, então, que houve um ganho sistêmico (a denominação ganho refere-se à expansão da oferta, produção e cobertura de serviços nos diferentes níveis de atenção à saúde - básica, média e alta complexidade ambulatorial e hospitalar) dos municípios em gestão plena, de 1998 a 2000, pois expandiu-se a abrangência de composição por níveis de assistência em quatro grupos e praticamente todos os grupos incorporaram a atenção básica.

Os condicionantes do desempenho dos municípios em GPSM podem ser vistos pela posição dos grupos na classificação estabelecida para aprendizado institucional, disponibilidade financeira e dimensão da máquina administrativa (Tabela 5).

Como mostra a Tabela 5, o alto nível de aprendizado institucional é auferido aos grupos 2 e 4, isto é, os municípios desses grupos passaram pelas habilitações mais qualificadas das NOB 93 e 96 . Os municípios que ofertam

Tabela 4

Resumo da variação do comportamento dos agrupamentos de municípios segundo os fatores gerados no estudo - 1998 e 2000 .

\begin{tabular}{|c|c|c|}
\hline Fator de destaque no grupo & 1998 & 2000 \\
\hline 1 (Atenção Básica) & - & - \\
\hline 2 (Alta complexidade) & $\begin{array}{l}\text { - Atenção básica }(75 \%)^{\star} \\
\text { - Capacidade de gestão hospitalar }(60 \%)^{\star}\end{array}$ & $\begin{array}{l}\text { - Atenção de média complexidade }(74 \%)^{\star} \\
\text { - Atenção básica }(79 \%)^{\star} \\
\text { - Capacidade de gestão hospitalar }(50 \%)^{*}\end{array}$ \\
\hline 3 (Capacidade hospitalar) & - Atenção de média complexidade $(52 \%)^{\star}$ & $\begin{array}{l}\text { - Atenção básica }(97 \%)^{\star} \\
\text { - Atenção média complexidade }(62 \%)^{\star}\end{array}$ \\
\hline 4 (Capacidade municipal hospitalar) & - & - Atenção básica $(90 \%)^{\star}$ \\
\hline 5 (Baixa capacidade de atenção e gestão) & - & - \\
\hline 6 (Média complexidade) & - & - Atenção básica (87\%)* \\
\hline
\end{tabular}

Fonte: Pesquisa Avaliação da Gestão Plena do Sistema Municipal, 2001.

* Percentual de municípios no grupo com avaliação alta no fator ou nível de assistência. 
Tabela 5

Classificação, em ordem crescente, dos grupos de municípios (1 a 6), segundo variáveis condicionantes, ano 2000.

\begin{tabular}{|c|c|c|c|c|c|c|}
\hline \multirow[t]{2}{*}{ Variáveis } & \multicolumn{6}{|c|}{ Ranking dos grupos } \\
\hline & $1 \underline{a}$ & $2 \underline{a}$ & 3ạ & $4 \underline{a}$ & $5 \underline{a}$ & $6 \underline{a}$ \\
\hline Alto aprendizado institucional & Grupos 2 e 4 & Grupo 6 & Grupo 3 & Grupo 1 & Grupo 5 & - \\
\hline Receita orçamentária per capita & Grupo 6 & Grupo 2 & Grupo 3 & Grupo 4 & Grupo 1 & Grupo 5 \\
\hline Capacidade de gasto & Grupo 6 & Grupo 3 & Grupo 4 & Grupos 1 e 2 & Grupo 5 & - \\
\hline $\begin{array}{l}\text { Gasto com pessoal nas despesas } \\
\text { municipais (\%) }\end{array}$ & Grupo 6 & Grupo 3 & Grupo 2 & Grupo 4 & Grupo 1 & Grupo 5 \\
\hline
\end{tabular}

Fonte: Pesquisa Avaliação da Gestão Plena do Sistema Municipal, 2001.

somente atenção básica ou que se destacam por um baixo desempenho em todos os níveis de assistência têm também os mais baixos percentuais de alto aprendizado institucional. Chama a atenção, portanto, como o aprendizado institucional acompanha o maior adensamento dos sistemas municipais de saúde.

Quanto à disponibilidade financeira (assimilada pela receita orçamentária per capita e capacidade de gasto) e à dimensão da máquina administrativa (assimilada pelo percentual de gasto com pessoal em relação às despesas totais do município), são os grupos formados por municípios médios (na classificação NESUR), seguidos dos municípios que agregam as capitais (grupo 2) que têm maior disponibilidade financeira e maiores gastos com pessoal, isto é, maior capacidade institucional (máquinas administrativas mais consistentes). Os grupos de pior desempenho ou com menor adensamento sistêmico, grupos 1 e 5, são justamente os que dispõem de menos recursos e menor capacidade institucional.

\section{Considerações finais}

Houve progresso positivo entre os municípios habilitados em GPSM, entre 1998 e 2000, no que se refere ao maior adensamento dos sistemas municipais de saúde ou ganho sistêmico e, portanto, uma melhoria nos padrões de oferta - capacidade instalada, produção e cobertura de serviços ambulatoriais e hospitalares -, financiamento e gasto federal nos diferentes níveis de atenção.

O ganho sistêmico é percebível tanto pelo progresso dos grupos de piores condições de oferta para os com melhores condições (medido pela expansão dos grupos com maiores médias de produção e cobertura em média complexidade - grupo 6 - e alta complexidade - grupo 2), quanto pela maior abrangência dos níveis de assistência interna aos grupos (maior número de combinações interníveis de assistência). Destaca-se que, em 2000, todos os grupos têm avaliação alta no fator que mede a capacidade de produção e cobertura da atenção básica, e dois grupos conseguem atingir alto grau de suficiência sistêmica, ou seja, obtêm valores altos em pelo menos três fatores.

$\mathrm{O}$ adensamento dos sistemas de saúde verificado nos municípios em GPSM não resulta, no entanto, necessariamente, em ampliação do acesso da população aos serviços de saúde existentes ou maior racionalidade sistêmica. Muito embora signifiquem melhores oportunidades de acesso e intensificação na utilização de serviços, não se identificou, na análise dos agrupamentos a população beneficiária das ações produzidas, a organização e o funcionamento da rede de saúde nestes municípios.

É interessante observar que as melhores condições de oferta estão associadas ao alto aprendizado institucional, maior autonomia gerencial, maior receita orçamentária, maior capacidade de gasto e maior gasto com pessoal, isto é, máquinas administrativas mais robustas. Dessa forma, o processo de descentralização da política de saúde - caracterizado pela forte indução federal, adesão dos municípios à GPSM baseada em critérios nacionais e condicionada à avaliação e decisão das instâncias de pactuação intergestores (CIB) - serviu como estímulo para o fortalecimento institucional desses municípios.

Mas esse perfil mais homogêneo do sistema de saúde, em 2000, não significa necessariamente uma melhoria nos padrões de eqüidade, considerando a eqüidade como a capacidade do sistema em oferecer mais recursos ( $l a-$ tu sensu) para os que mais necessitam de assistência. A despeito da melhoria da distribuição regional dos grupos (regiões mais pobres en- 
tram em grupos com melhores condições de oferta), permanecem graves distorções ainda entre os grupos.

Concluímos que a estratégia de descentralização induzida pela NOB 96 vem conseguindo fortalecer institucionalmente e aumentar as condições de oferta dos sistemas de saúde para os municípios habilitados em gestão plena. Contudo, permanece a questão da eqüidade em vista, sobretudo das condições impostas pelo contexto de restrição fiscal (grupos com piores condições de oferta dispõem de menos recursos fiscais) e a pesada herança de desigualdades econômicas e sociais do país.

\section{Referências}

DRAIBE, S. M., 1997. Avaliação da Descentralização das Políticas Sociais no Brasil: Saúde e Educação Fundamental. Informe Final do Projeto Estudios de Descentralización de Servicios Sociales, da División de Desarrollo Económico da Comisión Económica para América Latina y el Caribe. Campinas: Núcleo de Estudos de Políticas Públicas, Universidade Estadual de Campinas.

FREEMAN, R. \& MORAN, M., 2000. Reforming health care in Europe. In: Recasting European Welfare States (M. Ferrera \& M. Rhodes, ed.), pp. 35-58, London: Frank Cass.

HEIMANN, L. S., 1998. A Descentralização do Sistema de Saúde no Brasil - Uma Proposta de Investigação sobre o Impacto de Políticas. Relatório Final. São Paulo: Núcleo de Investigação em Serviços e Sistemas de Saúde, Instituto de Saúde, Secretaria de Estado da Saúde de São Paulo.

LEVCOVITZ, E.; LIMA, L. D. \& MACHADO, C. V., 2001. Política de saúde nos anos 90: Relações intergovernamentais e o papel das normas operacionais básicas. Ciência \& Saúde Coletiva, 6:269-318.

MS (Ministério da Saúde), 2001. Regionalização da Assistência à Saúde: Aprofundando a Descentralização com Eqüidade no Acesso (Norma Operacional da Assistência à Saúde. NOAS. SUS 01/01. Portaria MS/GM no 95, de 26 de Janeiro de 2001). Brasília: MS.

TEIXEIRA, S. M. F., 1990. Descentralização dos serviços de saúde: Dimensões analíticas. Revista de Administração Pública, 24:78-99.

VIANA, A. L. D., 1995. Modelos de Intervenção do Estado na Área de Saúde. Série Estudos de Saúde Coletiva 118. Rio de Janeiro: Instituto de Medicina Social, Universidade do Estado do Rio de Janeiro.

Recebido em 2 de maio de 2002

Versão final reapresentada em 19 de setembro de 2002

Aprovado em 14 de outubro de 2002 